\title{
THE
}

UNIVERSITY

University of Rhode Island

OF RHODE ISLAND

DigitalCommons@URI

\section{Theoretical Studies of the Energetics and Structures of Atomic Clusters}

J. Xie

University of Rhode Island

\section{J. A. Northby}

University of Rhode Island, jnorthby@uri.edu

David L. Freeman

University of Rhode Island, dfreeman@uri.edu

Jimmie D. Doll

Follow this and additional works at: https://digitalcommons.uri.edu/chm_facpubs

Terms of Use

All rights reserved under copyright.

\section{Citation/Publisher Attribution}

Xie, J., Northby, J. A., Freeman, D. L. \& Doll, J. D. (1989). Theoretical Studies of the Energetics and Structure of Atomic Clusters. Journal of Chemical Physics, 91(1), 612-619. doi: 10.1063/1.457447 Available at: http://dx.doi.org/10.1063/1.457447

This Article is brought to you for free and open access by the Chemistry at DigitalCommons@URI. It has been accepted for inclusion in Chemistry Faculty Publications by an authorized administrator of DigitalCommons@URI. For more information, please contact digitalcommons-group@uri.edu. 


\section{Theoretical studies of the energetics and structures of atomic clusters}

J. Xie, J. A. Northby, David L. Freeman, and J. D. Doll

Citation: J. Chem. Phys. 91, 612 (1989); doi: 10.1063/1.457447

View online: https://doi.org/10.1063/1.457447

View Table of Contents: http://aip.scitation.org/toc/jcp/91/1

Published by the American Institute of Physics

\section{Articles you may be interested in}

Noncrystalline structure of argon clusters. II. Multilayer icosahedral structure of ArN clusters $50<\mathrm{N}<750$

The Journal of Chemical Physics 84, 3491 (1986); 10.1063/1.450235

Structure and energetics of model metal clusters

The Journal of Chemical Physics 96, 8520 (1992); 10.1063/1.462305

The energetics and structure of nickel clusters: Size dependence

The Journal of Chemical Physics 94, 7376 (1991); 10.1063/1.460169

Icosahedral, decahedral, fcc, and defect-fcc structural models for ArN clusters, $\mathrm{N} \approx 500$ : How plausible are they?

The Journal of Chemical Physics 98, 4909 (1993); 10.1063/1.464946

Cluster size determination from diffractive He atom scattering

The Journal of Chemical Physics 105, 5408 (1996); 10.1063/1.472406

Structure and binding of Lennard-Jones clusters: $13 \leq \mathrm{N} \leq 147$

The Journal of Chemical Physics 87, 6166 (1987); 10.1063/1.453492

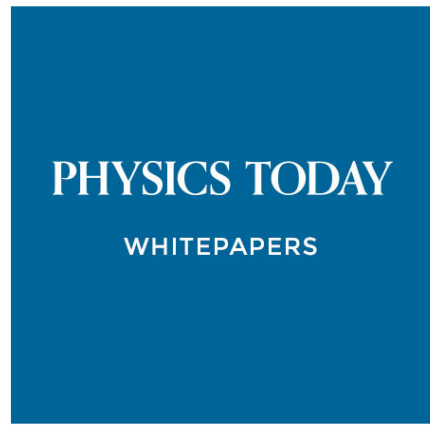

ADVANCED LIGHT CURE ADHESIVES

Take a closer look at what these environmentally friendly adhesive systems can do
READ NOW

PRESENTED BY

Q MASTERBOND' 


\title{
Theoretical studies of the energetics and structures of atomic clusters
}

\author{
J. Xie and J. A. Northby \\ Department of Physics, University of Rhode Island, Kingston, Rhode Island 02881 \\ David L. Freeman \\ Department of Chemistry, University of Rhode Island, Kingston, Rhode Island 02881 \\ J. D. Doll ${ }^{\text {a) }}$ \\ Los Alamos National Laboratory, MS G738, Los Alamos, New Mexico 87545
}

(Received 28 December 1988; accepted 24 March 1989)

\begin{abstract}
Comparative calculations of the binding energy and structure of relaxed closed-shell clusters of icosahedral and cuboctahedral point group symmetry are reported. The atoms are presumed to interact via either the Lennard-Jones or the Aziz-Chen (HFD-C) pair potential. The IC structure is found to be lower in total energy for less than 14 shells (10 179 atoms) in the Lennard-Jones case and for less than 13 shells ( 8217 atoms) in the HFD-C case. Detailed energetics are analyzed in order to elucidate the mechanism for the transition from icosahedral to cuboctahedral symmetry.
\end{abstract}

\section{INTRODUCTION}

One of the more interesting theoretical questions in the study of clusters is the properties of the minimal energy structures of finite systems interacting via short-range isotropic pair potentials. This question is motivated by the related experimental issue of the structure of free rare gas clusters. Small clusters are believed to be characterized by an icosahedrally derived noncrystalline symmetry. ${ }^{1}$ On the other hand, it is known that bulk rare gas crystals have a face centered cubic (FCC) crystalline symmetry. ${ }^{2}$ It is natural to ask at what cluster size the FCC structure becomes lower in energy than the icosahedral (IC) structure. Furthermore, it is of interest to determine the mechanisms that induce the transition from one to the other as the size of the system increases.

Experimental information on the transition from the finite IC behavior to the bulk FCC behavior is rather limited and consists primarily of electron diffraction studies of rare gas cluster beams. ${ }^{3-6}$ Using such methods, Farges, de Feraudy, Raoult, and Torchet ${ }^{3,4}$ inferred that the transition to the bulk-like structure occurs for systems larger than approximately 750 atoms. On the other hand, Lee and Stein, ${ }^{6}$ using similar techniques, found the transition to take place at approximately 1500 atoms. The exact relationship between these results and the lowest energy structure is somewhat unclear, since the cluster size distribution in a beam is relatively broad and known only approximately. Furthermore, even if the size were precisely known, the clusters in the beam would likely be distributed over a variety of bound state geometries. Since spectroscopic information on ground state structures of neutral rare gas clusters is currently unavailable, the determination of the structure of the lowest energy configuration is likely to remain a theoretical question for the near future.

To obtain a qualitative understanding of the origin of the increased stability of IC clusters in relation to FCC clusters, we note that the first complete shell surrounding a cen-

\footnotetext{
a) Present address: Department of Chemistry, Brown University, Providence, Rhode Island 02912.
}

tral atom in a close-packed lattice contains 12 atoms. Consequently, we examine the clusters of size 13 depicted in Figs. 1(a) and 1(b). In a 13 atom cluster the IC structure has 42 near-neighbor bonds whereas the FCC 13 atom cluster has 36 near-neighbor bonds. In order to form the additional near-neighbor bonds in the IC structure, the intershell bonds are slightly compressed from the distances at the interatomic potential minimum, and the intrashell bonds are slightly extended. ${ }^{7}$ As long as the force constant for the interatomic interactions is not too great, the resulting strains can be accommodated with little increase in the energy per bond. Consequently, the increased number of near neighbors significantly favors the IC structure. As described by Mackay, ${ }^{7}$ one can continue to add shells to the 13 atom IC structure to make increasingly large clusters while maintaining the same point group symmetry. The next shell of IC symmetry contains 42 atoms (for a total of 55) as shown in Fig. 1(c). In general, each additional shell contains $10 n^{2}+2$ atoms where $n$ is the shell number. This scheme leads to a series of closed shell structures having total numbers of atoms,

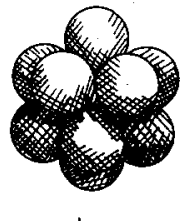

la

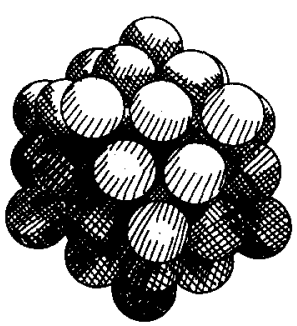

Ic

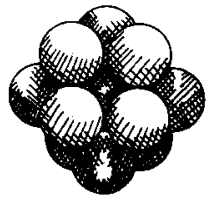

Ib

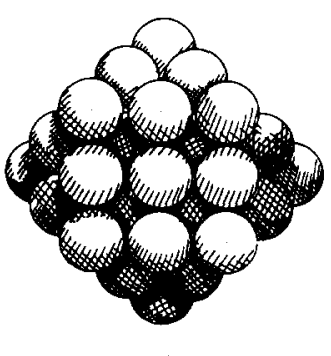

FIG. 1. Schematic representation of the first two closed-shell clusters of IC and FCC symmetry: (a) IC 13 atom cluster; (b) FCC 13 atom cluster; (c) IC 55 atom cluster, and (d) FCC 55 atom cluster. 
$N=13,55,147,309$, etc. In a similar manner we can form closed-shell clusters having cuboctahedral symmetry by taking an atom at the interior of an FCC bulk material and surrounding the atom by successively larger cooordination shells. The number of atoms per closed shell in a cuboctahedral structure (hereafter referred to as FCC) is the same as in the IC structures. An example is the 55 atom FCC structure depicted in Fig. 1(d). Although other cluster structures can be derived from bulk FCC and other close packed materials, to our knowledge the cuboctahedral-FCC scheme is the only one having the same number of atoms per closed shell as the IC structures.

The determination of the energy of relaxed closed shell structures of IC and FCC point group symmetry under the approximation that the atoms interact via a short-range isotropic pair potential has been considered by a number of previous workers. ${ }^{6,8,9}$ By relaxed, it is implied that the net force on each atom within the cluster is zero. A central question is which of the two structures (IC or FCC) is lower in energy as a function of the number of complete shells. Previous work has been limited to studies either using the standard Lennard-Jones (LJ) pair potential or truncated versions of the LJ model. Griffin and Andres ${ }^{8}$ utilized a truncated $\mathrm{LJ}$ potential and relaxed their initial configurations by a scale change. They found the FCC configuration to be lower in energy for systems having greater than approximately 1500 atoms. Lee and Stein ${ }^{6}$ used the full $\mathrm{LJ}$ potential but performed relaxations in the radial direction only. They found the transition to occur for systems having greater than about 3000 atoms. Most recently, Honeycutt and Andersen ${ }^{9}$ have performed complete relaxations out to seven shells $(N=1415)$, and incomplete relaxations out to 11 shells $(N=5083$ ). They concluded the transition to take place for systems larger than $\mathbf{5 0 0 0}$ atoms. To our knowledge, no one has ever actually found the crossing point between the IC and FCC structures, or, in fact, found any other structure that is lower in energy than a closed shell IC structure of the same size. Our goal is to find a definitive crossing point; i.e., to find a structure which is lower in energy than an IC cluster of the same size.

In this paper we investigate the energetics of clusters within a strictly classical model. A preliminary report of this work has appeared separately. ${ }^{10} \mathrm{We}$ are interested in the mathematical question of the location of the absolute minimum of a $3 N$-dimensional potential energy surface as a function of the number of atoms in the system $N$. Since the determination of the absolute minimum of a complex $3 N$-dimensional potential surface is not, in general, possible, we pose the more limited question of the relative energetics of fully relaxed clusters having IC and FCC symmetries. To further define the problem we have described the interactions between the atomic constituents using first the Lennard-Jones ( $\mathrm{LJ}$ ) model

$$
V_{\mathrm{LJ}}(r)=4 \epsilon\left[(\sigma / r)^{12}-(\sigma / r)^{6}\right]
$$

where $\epsilon$ is the well depth and $\sigma$ is the distance at which $V_{L J}(r)$ vanishes. We have then tested the sensitivity of the results to the form of the pair potential using a more realistic pair potential for argon (HFD-C) developed by Aziz and Chen. ${ }^{11}$ To our knowledge, this is the first time this realistic form of the pair potential has been used in cluster studies.

The contents of the remainder of this paper are as follows. We describe our method of determination of the relaxed structures in Sec. II. In Sec. III we present the results including both energetics and structural details of the relaxed clusters. We use this information to develop a qualitative understanding of the mechanism driving the transition from finite to bulk behavior. In Sec. IV we present our conclusions.

\section{METHOD}

In the current work, we have used the standard LJ model for the interatomic forces and have tested the sensitivity of the results to the form of the pair potential by using an improved potential introduced by Aziz and Chen. ${ }^{10}$ Our goal has been to determine and compare the energies of relaxed clusters having IC and FCC point group symmetries. Our procedure has been to begin with atomic coordinates placed on a regular Mackay icosahedral lattice ${ }^{7}$ and also on a regular FCC lattice. We then performed computer calculations to relax the structures until the force on each atom became insignificant. Finally, we checked that the relaxed structures obtained in this fashion maintained the original point group symmetry.

The method used for relaxation is a variant of the general class of gradient search methods called a "continued partan" method. ${ }^{12}$ It consists of an alternation of one-dimensional energy minimizations along a direction determined by the $N$-dimensional gradient, with an "acceleration step"; the acceleration step being a minimization along a direction obtained by connecting the results of the two previous greadient searches. Further details of the procedure along with the source code are presented elsewhere. ${ }^{13,14}$

As indicated, we have defined a structure to be completely relaxed when the forces on each atom vanish. In practice, we terminated the relaxations when the maximum force on any of the atoms was four orders of magnitude less than the maximum force after an initial relaxation step. Using this criterion, we found that the energy was stable to better than one part in $10^{6}$ for the largest structures and even more stable for the smaller structures. We investigated the stability of the minima obtained from the partan search by varying the coordinates randomly in a Monte Carlo fashion. ${ }^{15}$ The minima were found to be stable and no change in the calculated energies were obtained to the significance of the calculations.

\section{RESULTS}

\section{A. Lennard-Jones pair potential}

The results of the calculations using the $\mathbf{L J}$ model are given in Table I as a function of $n$, the number of shells, and $N$, the number of atoms in the cluster. In the table, $E_{b}$ is the binding energy in units of the LJ well depth $\epsilon$ and $\Delta E_{b}$ is the difference in energy between the two structures expressed in the same units. As discussed above, we believe the results to be accurate to better than the six significant figures present- 
TABLE I. The binding energy of icosahedral (IC) and cuboctahedral (FCC) Lennard-Jones clusters in units of the Lennard-Jones well depth.

\begin{tabular}{rrrrr}
\hline \hline$n$ & $N$ & $E_{b}(\mathrm{IC})$ & $E_{b}(\mathrm{FCC})$ & $\Delta E_{b}=E_{b}(\mathrm{FCC})-E_{b}(\mathrm{IC})$ \\
\hline 2 & 55 & 279.248 & 268.276 & -10.972 \\
3 & 147 & 876.461 & 854.376 & -22.085 \\
4 & 309 & 2007.21 & 1971.56 & -35.65 \\
5 & 561 & 3842.39 & 3792.09 & -50.30 \\
6 & 923 & 6552.72 & 6488.21 & -64.51 \\
7 & 1415 & 10308.8 & 10232.1 & -76.7 \\
8 & 2057 & 15281.5 & 15196.0 & -85.5 \\
9 & 2869 & 21641.3 & 21552.2 & -89.1 \\
10 & 3871 & 29558.9 & 29472.7 & -86.2 \\
11 & 5083 & 39204.8 & 39129.9 & -74.9 \\
12 & 6525 & 50749.8 & 50695.9 & -53.9 \\
13 & 8217 & 64364.4 & 64343.0 & -21.4 \\
14 & 10179 & 80219.3 & 80243.3 & +24.0 \\
\hline \hline
\end{tabular}

ed in Table I. Rigorously, the values given are only upper bounds (as they would be in any computer calculation). A graph of $\Delta E_{b}$ vs shell number is given in Fig. 2 (again in units of $\epsilon$ ). The maximum difference in energy between the two structures occurs at shell number 9, and we find FCC is lower in energy for the first time at shell number 14 $(N=10179)$. In Fig. 2, the solid line is obtained by first fitting $E_{b}$ for each structure to the same function used by Lee and Stein, ${ }^{6}$

$$
E_{b}=A+B N^{1 / 3}+C N^{2 / 3}+D N,
$$

where the coefficients are treated as free and independent parameters. The solid line is then calculated as the difference in the two fitting functions. The coefficients for the fit to each symmetry are given in Table II. The fit agrees with our calculated values to 0.1 energy units or better. The fit function predicts that a 14 shell FCC cluster is the first closed shell to be lower in energy than an IC cluster in agreement with the exact calculation. As a note of caution, the calculations make no predictions concerning the energies of structures of other symmetries but the same number of atoms. We cannot rule out the possibility that structures of other symmetries

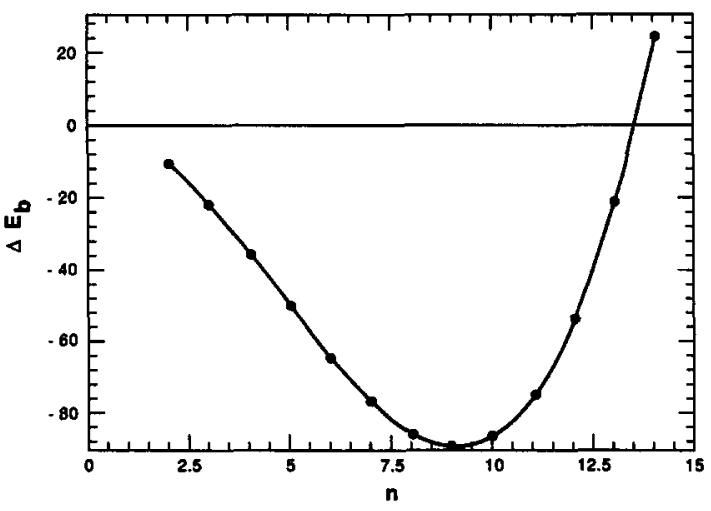

FIG. 2. The difference in binding energy $\Delta E_{b}$ between the cuboctahedral and icosahedral Lennard-Jones clusters as a function of shell number $n$. The energy is expressed in units of the Lennard-Jones well depth, the points are the calculated values given in Table $I$, and the solid line is obtained by fitting the results to Eq. (2). may be lower in energy than either IC or FCC for the cluster sizes we have studied. We have demonstrated that 13 shells are an upper bound to the size that an IC structure can be such that it has the possibility of being the lowest energy structure. We have also demonstrated that at 14 shells, FCC is lower in energy than IC. As an additional note of caution, nonclosed-shell values of $N$ when introduced into Eq. (2) should not be implied to have any meaning. Our calculations apply only to closed-shell values of $N$.

In continuum models the terms in Eq. (2) can be motivated as volume (linear in $N$ ) and surface contributions (proportional to $N^{2 / 3}$ ) with curvature dependent corrections (proportional to $N^{1 / 3}$ ). Again in continuum models, the constant term can be understood to account for the fact that when $N=1$, the binding energy is zero. In our atomistic calculations, we have not placed any physical significance on the coefficients. However, in the case of FCC clusters, the coefficient $D$ agrees well with the exact bulk binding energy per atom for an infinite $\mathrm{LJ}$ system $\left(8.61020 \epsilon^{16}\right)$. As a note of caution, the better than four figure agreement between the coefficient $D$ and the bulk binding energy is somewhat fortuitous. As we will discuss below, the binding energy of the central atom in a 14 shell FCC cluster is smaller than the bulk binding energy per atom in the fourth significant figure.

Beyond the question of the crossing point obtained by exploring the total energies of the clusters, it is of considerable interest to investigate the mechanism inducing the transition from IC to FCC behavior. Qualitatively, we might expect that FCC structures minimize bulk energy contributions and IC structures minimize the surface contribution but at the price of increased energy in the interior of the cluster. As the size of the clusters increase, the interior con-

TABLE II. The coefficients for the fitting function [Eq. (2)] for icosahedral (IC) and cuboctahedral (FCC) Lennard-Jones clusters $(n=2-14)$.

\begin{tabular}{lccccc}
\hline \hline & & & $B$ & $C$ & $D$ \\
\hline IC & 9.8248958 & 1.5534957 & -14.217539 & 8.5326356 \\
FCC & 4.4050797 & 5.8929195 & -16.046618 & 8.6106745 \\
\hline \hline
\end{tabular}


tributions will begin to dominate and the FCC structure will eventually be favored.

To test the extent to which our expectations are realized we can examine in greater detail where the contributions to the energy arise. For the next level of detail, we have found it convenient to define the binding energy per atom for atom $i$

$$
E_{b i}=-\frac{1}{2} \sum_{j}^{\prime} V_{\mathrm{LJ}}\left(r_{i j}\right)
$$

where $r_{i j}$ is the distance between the $i$ th and $j$ th atoms and the prime on the summation indicates that the term $j=i$ is to be excluded. The total binding energy is then given by $E_{b i}$ summed over all atoms in the cluster. We then define the average binding energy per atom of clusters of size $n$ in shell $m$ ( $m$ runs from $m=0$ to $n$ ), $E_{s h}(n, m)$ by

$$
E_{s h}(n, m)=\left[1 /\left(10 m^{2}+2\right)\right] \sum_{i} E_{b i}
$$

where the summation in Eq. (4) is over the $10 m^{2}+2$ atoms in shell $m$. Graphs of $E_{s h}(n, m)$ are given in Fig. 3 for FCC (the open circles) and IC (the filled circles). As in Fig. 2, the energy is expressed in units of $\epsilon$. It is evident that as $n$ increases, $E_{s h}(n, m)$ for the interior shells of FCC clusters rapidly approaches its asymptotic bulk value. Deviations from the bulk value are only apparent in the outermost three shells. In contrast, for the IC clusters, $E_{s h}(n, m)$ is a rather sensitive function of both $n$ and $m$. The most dramatic variations occur for the innermost shells where the binding energy per atom becomes increasingly small as $n$ increases. This indicates that there is significant compression of the interior of a large IC cluster which becomes greater as the cluster size increases. In contrast to FCC clusters, there is no asymptotic value to the interior energy. Evidently, the central atom is compressed significantly into the repulsive region of the $\mathrm{LJ}$ interaction potential. This compression has also been noted in the work of Farges et al. ${ }^{17}$ In the interior, $E_{s h}(n, m)$ is lower for IC than for FCC until the third shell from the surface, at which point they become approximately equal. It is only for the outer most two shells that $E_{s h}(n, m)$ becomes lower for FCC than IC. The difference in $E_{s h}(n, n)$ at the outermost shell is approximately $4 \%$. Although the difference in energy is quite small on a per atom basis, the fraction of the total number of atoms in the surface shell for a 13 shell cluster is approximately $20 \%$. Consequently, the contribution to the total binding energy coming from the surface layer is quite large and sufficient to render the IC configuration lower in energy than FCC. It is quite striking that the surface profile of $E_{s h}(n, m)$ is insensitive to the size of the cluster; i.e., $E_{s h}(n, n)$ and $E_{s h}(n, n-1)$ are nearly independent of $n$.

A more detailed picture of the origins of the energy contributions can be found by plotting $E_{b i}$ for each atom as a function of the distance $R$ of the atom from the central atom in the cluster. The results are shown in Fig. 4 for cluster sizes $n=8,11$, and 14. The distances in Fig. 4 are expressed in units of the distance to the Lennard-Jones potential minimum $\left[2^{1 / 6} \sigma\right]$ and the energy is expressed in units of $\epsilon$. Although fourteen shell clusters have 10179 atoms, the high symmetry of the relaxed structures renders most of the
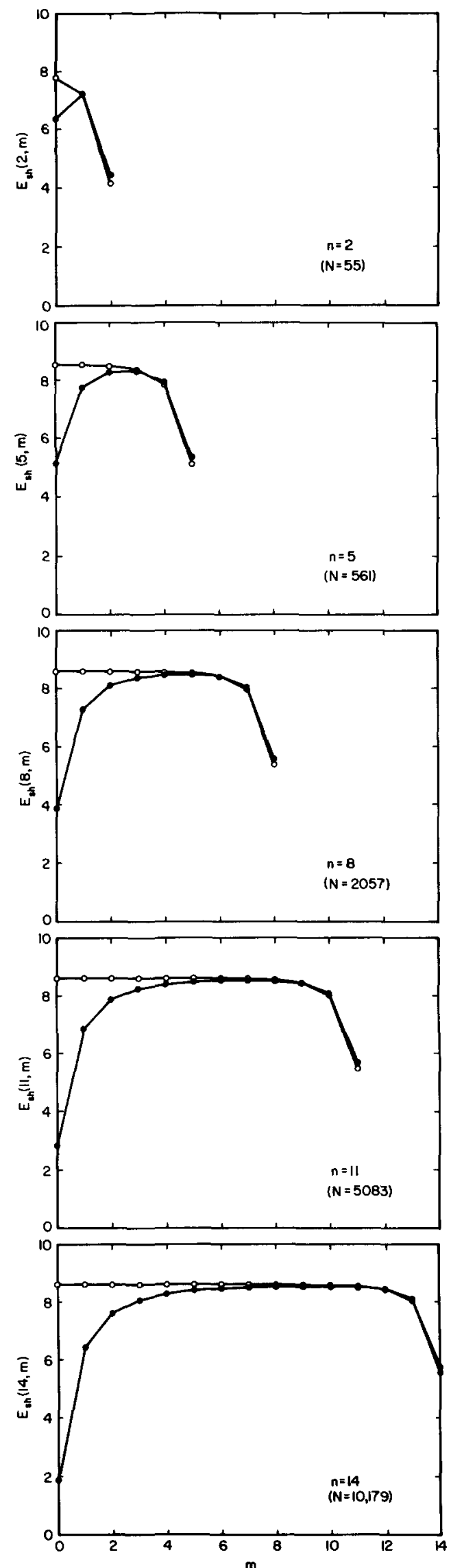

FIG. 3. Average binding energy per atom within a shell [see Eq. (4)] as a function of shell number $\boldsymbol{m}$ and total number of shells in the cluster, $n$ for Lennard-Jones clusters. The energy is expressed in units of the LennardJones well depth. The open circles are the FCC results and the closed circles are the IC results. 

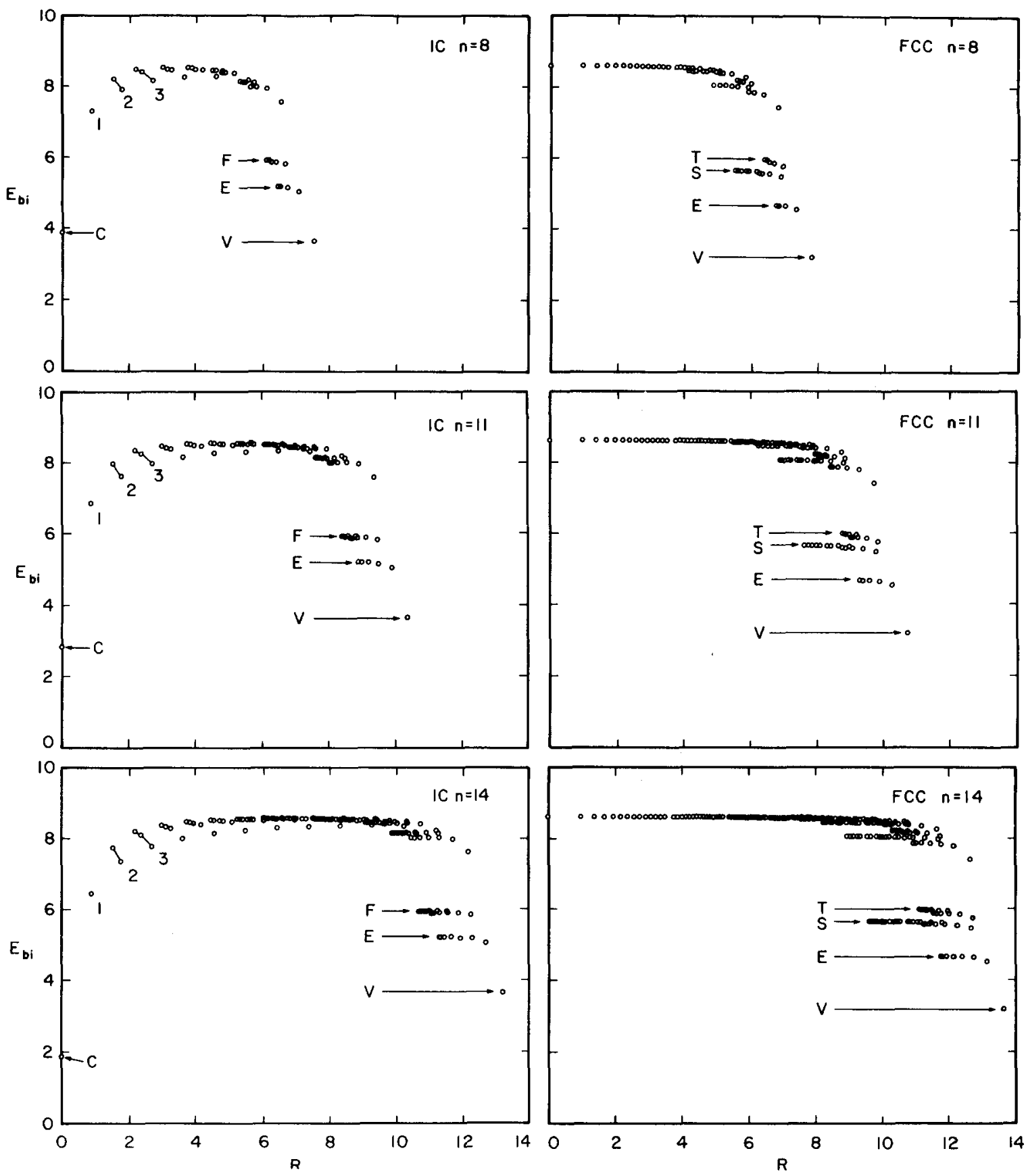

FIG. 4. Binding energy per atom $E_{b i}$ as a function of the distance from the center of the cluster, $R$ and the number of shells, $n$ for both IC and FCC LennardJones clusters. The additional symbols are defined in the text. The energy is expressed in units of the Lennard-Jones well depth and the distance in units to the Lennard-Jones minimum.

points coincident. This is clearly evident in the figure. In Fig. 4 for the IC structures, we have labeled the central atom with a " $C$," and the first shell (consisting of 12 equivalent vertex atoms) by a " 1 ." The second shell, labeled by a " 2 ," consists of two distinct kinds of atoms; 12 vertex atoms and 30 edge atoms. We have connected the two points associated with the second shell by a line for clarity. The vertex atoms are less tightly bound than the edge atoms by approximately $0.3 \epsilon$. The third shell, labeled by a "3" and again connected with a line for clarity, consists of three kinds of atoms; 12 vertex atoms, 60 edge atoms, and 20 face atoms. The binding energy of the vertex atoms is approximately $0.2 \epsilon$ smaller than the edge atoms. The edges atoms are approximately $0.1 \epsilon$ smaller in binding energy than the face atoms. This pattern continues for the remaining interior shells. As we continue outward the binding energy differences between the various sites becomes increasingly small until we reach the surface. At the surface, we can see a strong distinction in binding energy between the three classes of atoms. We have labeled the surface vertex atoms by a " $V$," the surface edge atoms by an " $E$ " and the surface face atoms by an " $F$." There is a small variation in binding energy within the surface edge 
atoms and within the surface face atoms, which is responsible for the slight curvature observed. The difference in binding energy between the vertex, edge, and face atoms can be understood in terms of the difference in the number of nearest neighbors associated with each site. In a large IC structure, face atoms have nine nearest neighbors, edge atoms have eight nearest neighbors, and vertex atoms have only six nearest neighbors. This pattern is reflected in the binding energies observed in Fig. 4. From the IC figures it is evident that some atoms not at the surface of the larger clusters are further from the origin than some of the surface atoms. This distance relationship is an obvious consequence of the geometry of the cluster. That such atoms are not surface atoms is evident by their significantly larger binding energy. To avoid confusion, only the surface atoms have been labeled with an $\mathrm{F}, \mathrm{E}$, or $\mathrm{V}$.

As we observed in Fig. 3, as we move inward from the surface of an FCC cluster, the binding energy rapidly approaches a constant. This constant is nearly the bulk binding energy per atom in a bulk FCC LJ solid (8.610 20 $\epsilon$ ). This limiting behavior is again evident in Fig. 4. In particular, the binding energy of the central atom in clusters of size 8,11 , and 14 is $8.5894 \epsilon, 8.6023 \epsilon$, and $8.6061 \epsilon$, respectively. As a consequence of the rapid approach to the bulk value at the interior of FCC clusters, no shell structure is apparent in the figure in contrast to the IC case.

In a FCC cluster there are two kinds of faces (triangular and square) as can be seen in Fig. 1. Consequently, we expect to see four broad classes of atoms at the surface of an FCC cluster. In particular, the surface atoms can be classified into vertex, edge, triangular face, and square face atoms. These categories are mirrored in Fig. 4, where we have labeled the triangular face atoms with a "T," the square face atoms with an "S," the edge atoms with an $E$ and the vertex atoms with a V. The energy differences associated with the four kinds of surface atoms arise because triangular face atoms have nine nearest neighbors, square face atoms have eight nearest neighbors, edge atoms have seven nearest neighbors, and vertex atoms have five nearest neighbors. This qualitative ordering of energies is apparent in the figure. As in the IC case, some interior atoms are further from the center than some of the surface atoms. That these atoms are in the interior is clear from their higher binding energies.

As noted by others, ${ }^{6,17}$ while the atoms in a relaxed IC cluster have icosahedral point symmetry, the edges and faces exhibit a slight curvature. We have found the curvature to be rather small and have displayed the effect for an edge in Table III. In the table we list the atoms along the edge of a 14 shell IC cluster such that we number them in sequence with atoms 1 and 15 at any two adjacent vertices and atom 8 at the midpoint of the edge. In Table III, $R^{*}(I)$ is the distance of atom $I$ in a relaxed IC cluster from the central atom in units of the distance to the atom at the midpoint of the edge (atom 8 in the Table). To demonstrate the curvature we compare $R^{*}(I)$ to the same quantity, $R_{M}^{*}(I)$, calculated along the straight edge of a regular Mackay icosahedron. The curvature is most apparent by displaying the ratio $R_{M}^{*}(I) / R^{*}(I)$. The small deviations indicate a very slight but smooth outward curvature. In Table IV we display a
TABLE III. The distance between the central atom and the atoms along the edge of a 14 shell IC cluster in units of the distance between the central atom and the atom at the midpoint of the edge.

\begin{tabular}{rccc}
\hline$I$ & $R^{*}(\mathrm{I})$ & $R_{M}^{*}(\mathrm{I})$ & $R^{*}(\mathrm{I}) / R_{M}^{*}(\mathrm{I})$ \\
\hline 1 & 1.16775490 & 1.17557050 & 0.99335165 \\
2 & 1.12494327 & 1.13164839 & 0.99407490 \\
3 & 1.08840228 & 1.09310595 & 0.99569696 \\
4 & 1.05759378 & 1.06052987 & 0.99723149 \\
5 & 1.03289879 & 1.03448394 & 0.99846769 \\
6 & 1.01479618 & 1.01547078 & 0.99933568 \\
7 & 1.00372657 & 1.00389005 & 0.99983716 \\
8 & 1.00000000 & 1.00000000 & 1.00000000 \\
9 & 1.00372908 & 1.00389005 & 0.99983965 \\
10 & 1.01480014 & 1.01547078 & 0.99933958 \\
11 & 1.03290534 & 1.03448394 & 0.99847402 \\
12 & 1.05760161 & 1.06052987 & 0.99723888 \\
13 & 1.08841175 & 1.09310595 & 0.99570563 \\
14 & 1.12495412 & 1.13164839 & 0.99408449 \\
15 & 1.16776768 & 1.17557050 & 0.99336252 \\
\hline \hline
\end{tabular}

similar analysis of the edge atoms in the FCC cluster. In the table, $R_{C}^{*}(I)$ is the distance from the center of the atoms along the straight edge of a regular unrelaxed FCC cluster in units of the distance to atom 8. In the FCC case, the edge is curved but in a more complex fashion than in the IC case. The vertex atoms are displaced outward with respect to a tangent line at atom 8 whereas the atoms in the vicinity of atom 8 are displaced inward. It is notable that the symmetry along the edge is maintained to at least nine significant figures for the FCC cluster and to seven significant figures for the IC cluster. This difference between IC and FCC is a reflection of a general observation that $F C C$ relaxed with a smaller number of iterations than IC for the properties we studied. We believe this improved convergence rate is a result of the lack of strain in the interior of an FCC cluster.

\section{B. The HFD-C pair potential}

The $\mathrm{LJ}$ potential discussed in the previous subsection is commonly used in computer simulations because of its ease

TABLE IV. The distance between the central atom and the atoms along the edge of a 14 shell FCC cluster in units of the distance between the central atom and the atom at the midpoint of the edge.

\begin{tabular}{rccc}
\hline \multicolumn{1}{c}{$I$} & $R *(I)$ & $R *(I)$ & $R *(I) / R *(I)$ \\
\hline 1 & 1.15630297 & 1.15470052 & 1.00138776 \\
2 & 1.11592402 & 1.11574994 & 1.00115602 \\
3 & 1.08169373 & 1.08169682 & 0.99999714 \\
4 & 1.05298387 & 1.05301639 & 0.99996911 \\
5 & 1.03013114 & 1.03015750 & 0.99997441 \\
6 & 1.01350219 & 1.01351413 & 0.99998822 \\
7 & 1.00339225 & 1.00339560 & 0.99999666 \\
8 & 1.00000000 & 1.00000000 & 1.00000000 \\
9 & 1.00339225 & 1.00339560 & 0.99999666 \\
10 & 1.01350219 & 1.01351413 & 0.99998822 \\
11 & 1.03013114 & 1.03015750 & 0.99997441 \\
12 & 1.05298387 & 1.05301639 & 0.99996911 \\
13 & 1.08169373 & 1.08169682 & 0.99999714 \\
14 & 1.11592402 & 1.11574994 & 1.00015602 \\
15 & 1.15630297 & 1.15470052 & 1.00138776 \\
\hline \hline
\end{tabular}


in evaluation rather than its accuracy with respect to experiment. The sensitivity of any conclusions of a numerical simulation to the form of the pair potential used is always a concern. We have tested the sensitivity of our results to the precise form of the pair potential by performing energy relaxations of clusters using the potential (HFD-C) developed by Aziz and Chen ${ }^{11}$ and parametrized for argon,

$$
\begin{aligned}
V_{\text {HFD-C }}(r)= & a r^{\gamma} \exp (-\alpha r) \\
& -\left(C_{6} / r^{6}+C_{8} / r^{8}+C_{10} / r^{10}\right) F(r),
\end{aligned}
$$

where

$$
F(r)= \begin{cases}\exp \left[-(r / d-1)^{2}\right], & r<d \\ 1, & r \geqslant d .\end{cases}
$$

In Eqs. (5) and (6) a, $\gamma, \alpha, C_{6}, C_{8}, C_{10}$, and $d$ are constants whose values are given in Ref. 11 . This potential has a larger force constant in the vicinity of the minimum than the $\mathrm{LJ}$ potential, and has a somewhat larger well depth (143.2 K vs $119.8 \mathrm{~K}$ for the $\mathrm{LJ}$ potential). It is believed that the HFD-C potential is a significantly more accurate representation of the argon-argon interaction than the $\mathrm{LJ}$ model. In the calculations with the HFD-C potential, our goal was to determine whether the crossing point was qualitatively altered by this more realistic potential. Since we found that Eq. (2) fit the LJ results to high accuracy, we felt confident that a similar fit to fewer data points would be adequate to predict the crossing in the HFD-C case. Consequently, we relaxed structures within the HFD-C model for only shell numbers $n=1-7$ and 10 to determine the fit coefficients which are listed in Table $\mathbf{V}$. The results of the relaxation are given in Table VI and plotted in Fig. 5. The units of energy in Table VI and Fig. 5 are the HFD-C well depth. The fit predicts that the minimum occurs at approximately eight shells and the FCC becomes lower in energy at the thirteenth shell. To check this prediction we relaxed FCC and IC clusters within the HFD-C model for a 13 shell cluster. The exact 13 shell results is also given in Table VI and Fig. 5. The agreement between the extrapolated fit function and the full calculation is excellent. The crossing at the 13th shell is in qualitative, although not quantitative, agreement with the $\mathrm{LJ}$ results. This agreement is a measure of the insensitivity of the conclusion to the exact form of the pair potential. As a sidelight, it is interesting to note that while the FCC structure is unstable and collapses into the IC in the $\mathrm{LJ}$ case for a 13 atom cluster, the FCC 13 atom structure is stable with the HFD$\mathrm{C}$ potential. We have not performed the same detailed structural analysis in the HFD-C case that we performed for the $\mathrm{LJ}$ results, because the energetics are so similar.

TABLE V. The coefficients for the fitting function [Eq. (2)] for icosahedral (IC) and cuboctahedral (FCC) HFD-C clusters $(n=1-7,10)$.

\begin{tabular}{lccccc}
\hline \hline & $A$ & $B$ & $C$ & $D$ \\
\hline IC & 4.9594809 & 1.1613538 & -11.592934 & 7.6721252 \\
FCC & 3.0731707 & 3.6507413 & -13.154471 & 7.7466403 \\
\hline
\end{tabular}

TABLE VI. The total binding energy of icosahedral (IC) and cuboctahedral (FCC) HFD-C clusters in units of the well depth (143.2 K).

\begin{tabular}{rrccc}
\hline \hline$n$ & \multicolumn{1}{c}{$N$} & $E_{b}(\mathrm{IC})$ & $E_{b}(\mathrm{FCC})$ & $\begin{array}{c}\Delta E_{b}=E_{b}(\mathrm{FCC}) \\
-E_{b}(\mathrm{IC})\end{array}$ \\
\hline 1 & 13 & 43.2426 & 39.5968 & -3.6458 \\
2 & 55 & 263.838 & 252.844 & -10.944 \\
3 & 147 & 816.021 & 794.713 & -21.308 \\
4 & 309 & 1853.56 & 1820.20 & -33.36 \\
5 & 561 & 3529.95 & 3484.23 & -45.72 \\
6 & 923 & 5998.63 & 5941.74 & -56.89 \\
7 & 1415 & 9412.99 & 9347.62 & -65.37 \\
10 & 3871 & 26863.9 & 26804.6 & -59.3 \\
13 & 8217 & 58347.3 & 58373.6 & +26.3 \\
\hline \hline
\end{tabular}

\section{CONCLUSIONS}

We have found that closed shell IC structures are lower in energy than closed shell FCC structures for considerably larger size clusters than have previously been inferred either experimentally ${ }^{3-6}$ or theoretically. ${ }^{6,8,9}$ It is important to recognize that these results say nothing about crossings of IC and FCC based geometries for incomplete shell clusters. Indeed, Honeycutt and Andersen ${ }^{9}$ have implied that such nonclosed-shell crossing may occur earlier than we have found for closed shell clusters. The results also do not make any statements concerning clusters of other symmetries that may be still lower than those considered here. Bulk LJ solids have hexagonal close packed (HCP) symmetry, and HCP clusters may prove to be lower at a smaller cluster size within the $\mathrm{LJ}$ model. We restate that we have chosen to investigate FCC rather than HCP, because FCC clusters contain the same number of atoms in a closed shell as IC, whereas HCP clusters do not. Direct comparisons between closed-shell HCP and IC are ambiguous. We can state that within the LJ model, an upper bound to the size that a closed-shell IC cluster can be and still be the minimal energy structure is 13 .

We have found that FCC clusters approach bulk behav-

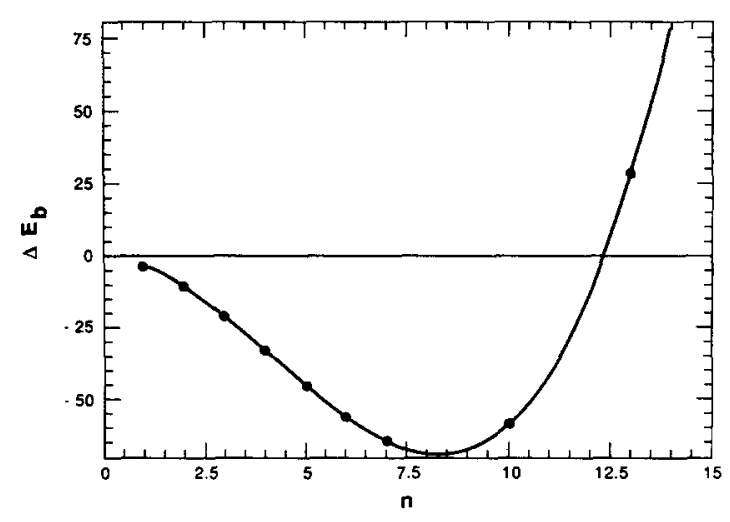

FIG. 5. The difference in binding energy $\Delta E_{b}$ between the cuboctahedral and icosahedral HFD-C clusters as a function of shell number $n$. The energy is expressed in units of the HFD-C well depth, the points are the calculated values given in Table VI, and the solid line is obtained by fitting the results to Eq. (2). 
ior in the interior rather rapidly with respect to cluster size. In contrast, IC clusters contain a strained high energy interior region, but the surface atoms have a higher binding energy than FCC structures. Since small clusters are dominated by surface atoms, the higher binding energy in the surface region for IC clusters favors them over FCC. For larger clusters the ratio of the number of interior atoms to surface atoms increases, and the more favorable interior structure of FCC clusters becomes the dominating factor. ${ }^{18}$

By ignoring three-body and higher-order contributions from the potential model, we have neglected directional contributions which may qualitatively change the nature and location of the crossing. Furthermore, the precise energetics will be influenced by the quantum zero point motion of the atoms in the clusters. Consequently, extrapolation of our conclusions to real systems must be treated with caution.

After this work was completed, we became aware of the results of van de $\mathrm{Waal}^{19}$ who performed relaxation studies of IC and FCC clusters for $n=1-11$ within the $\mathrm{LJ}$ model. His reported binding energies are in complete agreement with Table I for these cluster sizes. In addition, van de Waal used a two term fit function to extrapolate to the closed shell cluster size where the binding energy of the IC structure first becomes lower than the FCC structure. His prediction of $n=14$ is in agreement with the complete relaxation studies reported here. As discussed by van de Waal, the cluster size at which the binding energy of IC based structures becomes lower than the binding energy of FCC based structures may be significantly smaller if nonclosed-shell clusters are considered. This expectation is based on the realization that cuboctahedral FCC clusters have a rather unfavorable surface energy. For atomistic clusters based on FCC symmetries, the surface energy may be minimized by bounding the cluster with a Wulff polyhedron. ${ }^{20}$ Using this Wulff construction, van de Waal estimated the crossing to occur between 2000 and 3000 atoms. As mentioned above, however, com- parison of the energetics of clusters of differing $N$ on a per atom basis can lead to ambiguities.

\section{ACKNOWLEDGMENTS}

D.L.F. acknowledges the Donors of the Petroleum Research Fund, administered by the American Chemical Society and Research Corporation for partial support of this work. J.A.N. acknowledges the National Science Foundation (DMR 8405190) for partial support of this work. Acknowledgment is also made to the University of Rhode Island Academic Computer Center for additional support.

'M. R. Hoare, Adv. Chem. Phys. 40, 49 (1979).

${ }^{2}$ N. W. Ashcroft and N. D. Mermin, Solid State Physics (Saunders College, Philadelphia, 1976).

${ }^{3} J$. Farges, M. F. de Feraudy, B. Raoult, and G. Torchet, J. Chem. Phys. 78, 5067 (1983).

${ }^{4} J$. Farges, M. F. de Feraudy, B. Raoult, and G. Torchet, J. Chem. Phys. 84, 3491 (1986).

${ }^{5}$ S. S. Kim and G. D. Stein, J. Colloid Interf. Sci. 87, 180 (1982).

6J. W. Lee and G. D. Stein, J. Phys. Chem. 91, 2450.(1987).

${ }^{7}$ A. L. Mackay, Acta Cryst. 15, 916 (1962).

${ }^{8}$ G. L. Griffin and R. P. Andres, J. Chem. Phys. 71, 2522 (1979).

${ }^{9} J$. D. Honeycutt and H. C. Andersen, J. Phys. Chem. 91, 4950 (1987).

${ }^{10} \mathrm{~J}$. A. Northby, J. Xie, D. L. Freeman, and J. D. Doll, Z. Phys. D (in press).

"R. A. Aziz and H. H. Chen, J. Chem. Phys. 67, 5719 (1977).

${ }^{12}$ D. A. Pierre, Optimization Theory with Applications (Wiley, New York, 1969).

${ }^{13}$ J. A. Northby, J. Chem. Phys. 87, 6166 (1987).

${ }^{14} \mathrm{~J}$. Xie, M. S. thesis, University of Rhode Island, 1988.

${ }^{15}$ N. Metropolis, A. W. Rosenbluth, M. N. Rosenbluth, A. H. Teller, and E. Teller, J. Chem. Phys. 21, 1087 (1953).

${ }^{16} \mathrm{C}$. Kittel, Introduction to Solid State Physics, 4th ed. (Wiley, New York, 1971), Chap. 3.

${ }^{17}$ J. Farges, M. F. de Feraudy, B. Raoult, and G. Torchet, Acta Cryst. A 38, 656 (1982).

${ }^{18} \mathrm{~A}$ similar rational within a continuum description has been advanced by P. M. Ajayan and L. D. Marks, Phys. Rev. Lett. 60, 585 (1988).

${ }^{19}$ B. W. van de Waal, J. Chem. Phys. 90, 3407 (1989).

${ }^{20}$ S. Ino, J. Phys. Soc. Jpn. 27, 941 (1969). 